\title{
Meta-análisis preliminar de ensayos clínicos aleatorizados de ivermectina para tratar la infección por SARS-CoV-2
}

\author{
Preliminary meta-analysis of randomised clinical trials of ivermectin to treat SARS-CoV-2 infection
}

\section{Comentado de:}

Hill $A$, et al. Research Square 2021:10.21203/rs.3.rs$148845 / v 1^{1}$

\section{Introducción}

La ivermectina es un fármaco antiparasitario establecido con licencia desde 1981 y más recientemente aprobado por sus efectos antiinflamatorios contra la rosácea. Está siendo investigado para su utilización contra el SARS-CoV-2.

\section{Objetivo}

Evaluar la evidencia disponible de ensayos controlados aleatorizados (ECA) sobre la eficacia de la ivermectina en pacientes con COVID-19.

\section{Métodos}

Revisión sistemática y meta-análisis. Se realizó una búsqueda sistemática en PUBMED, EMBASE, MedRxiv y registros de ensayos clínicos en Diciembre de 2020 y se contactó a los autores de los ensayos no publicados. Se excluyeron los estudios de prevención. Los datos fueron extraídos y verificados de forma cruzada por dos revisores. El desenlace primario fue la mortalidad por todas las causas desde la aleatorización hasta el final del seguimiento. Otros desenlaces fueron los cambios en los marcadores inflamatorios, la supresión viral, la recuperación clínica y la duración de la hospitalización. Se realizó un meta-análisis para la mortalidad por todas las causas, por intención de tratar, de todos los pacientes aleatorizados. Para el análisis de mortalidad se incluyeron los ensayos con al menos dos muertes informadas. Los efectos se expresaron como razón de riesgo (RR) con sus intervalos de confianza (IC) del $95 \%$ para los resultados binarios y se aplicó una corrección de continuidad de 0,5 a los brazos de tratamiento sin muertes. La heterogeneidad fue evaluada por $\mathrm{I}^{2}$. Se evaluó el riesgo de sesgo de todos los estudios incluidos mediante la herramienta Cochrane de 2011

\section{Resultados}

Se incluyeron 18 ECA (cinco publicados, seis preimpresiones, seis comunicaciones de resultados no publicados y uno con datos recuperados de la web del registro de ensayos clínicos; 12 de los cuales eran simple o doble-ciego y seis, abiertos) involucrando 2.282 pacientes en total y un rango de 24 a 400 participantes. De los 18 ensayos, 11 fueron de considerados de mala calidad, cuatro de calidad regular y sólo tres de alta calidad. La mitad de los ECA investigó la ivermectina como dosis única y el resto, con dosificación de hasta siete días. En general (11 ECA) se evaluó la ivermectina en participantes con COVID-19 leve a moderado.

Cuatro de seis ECA mostraron un tiempo de recuperación clínica significativamente más rápido en los pacientes que recibieron ivermectina (aproximadamente 1 día menos) y en los seis ECAs que lo evaluaron, en la rama asignada de esta droga se documentó una duración significativamente más corta de las hospitalizaciones en comparación con el grupo control.

Seis ECA evaluaron la mortalidad en una diversidad de comparaciones, esquemas y poblaciones (Tabla 1 ). Se observó una reducción del $75 \%$ en la mortalidad (RR 0,25 [IC 95\% 0,12 a $0,52] ; p=0,0002 ; I^{2} 34 \%$; Figura 1).

El riesgo de sesgo en cuanto a la falta de ceguera de los estudios fue corregido de acuerdo a los autores, pues la objetividad del resultado muerte implica bajo riesgo de sesgo si se realiza una evaluación ciega del desenlace (improbablemente influenciada por el conocimiento de la intervención asignada), a diferencia del cegamiento del personal y de los participantes, que podría implicar una posibilidad de desviaciones del protocolo o la realización de co-intervenciones de manera no balanceada en los grupos del estudio.

Tabla 1. Tabla de resumen de las características de los estudios incluidos en el análisis de la mortalidad en ensayos clínicos aleatorizados de ivermectina para el tratamiento de COVID-19. Notas: Iv: ivermectina; Dox: doxiciclina; CU: cuidados usuales; HCQ: hidroxicloroquina; HFA: hidroxicloroquina, favipiravir y azitromicina

\begin{tabular}{|c|c|c|c|c|c|c|c|c|c|}
\hline $\begin{array}{l}\text { Autor } \\
\text { (año) }\end{array}$ & País & $\mathbf{N}$ & Cegamiento & Dosis, días & Pacientes & Intervención & $\begin{array}{l}\text { Mortalidad } \\
\text { en el gru- } \\
\text { po inter- } \\
\text { vención }\end{array}$ & Comparador & $\begin{array}{l}\text { Mortalidad } \\
\text { en el gru- } \\
\text { po com- } \\
\text { paración }\end{array}$ \\
\hline $\begin{array}{l}\text { Hashim } \\
(2020)^{2}\end{array}$ & Iraq & 140 & Simple ciego & $\begin{array}{c}0,2 \text { a } 0,4 \\
\mathrm{mg} / \mathrm{kg} 2 \text { a } 3 \\
\text { días }\end{array}$ & Sintomáticos & $\begin{array}{c}\text { Iv }+ \text { Dox }+ \\
\text { CU }\end{array}$ & $3 \%$ & $\mathrm{CU}$ & $9 \%$ \\
\hline $\begin{array}{l}\text { Mahmud } \\
(2020)^{3}\end{array}$ & Bangladesh & 363 & Doble ciego & $\begin{array}{l}0,2 \mathrm{mg} / \mathrm{kg}, 1 \\
\text { día }\end{array}$ & $\begin{array}{c}\text { Leves a } \\
\text { moderados }\end{array}$ & $\begin{array}{c}\mathrm{Iv}+\mathrm{Dox}+ \\
\mathrm{CU}\end{array}$ & $0 \%$ & $\mathrm{CU}$ & $2 \%$ \\
\hline $\begin{array}{l}\text { Niaee } \\
(2021)^{4}\end{array}$ & Irán & 180 & Doble ciego & $\begin{array}{l}0,2 \mathrm{mg} / \mathrm{kg} 1 \mathrm{a} \\
3 \text { días }\end{array}$ & $\begin{array}{c}\text { Leves a } \\
\text { moderados }\end{array}$ & $\mathrm{IV}+\mathrm{CU}$ & $3 \%$ & $\begin{array}{c}\text { Placebo + } \\
\text { CU }\end{array}$ & $18 \%$ \\
\hline $\begin{array}{l}\text { Kirti } \\
(2021)^{5}\end{array}$ & India & 112 & Doble ciego & 12 mg, 5 días & $\begin{array}{l}\text { Leves a } \\
\text { moderados }\end{array}$ & $\mathrm{IV}+\mathrm{CU}$ & $0 \%$ & $\begin{array}{c}\text { Placebo + } \\
\text { CU }\end{array}$ & $7 \%$ \\
\hline $\begin{array}{l}\text { Elgazzar } \\
(2020)^{6}\end{array}$ & Egipto & 400 & Abierto & $\begin{array}{l}0,4 \mathrm{mg} / \mathrm{kg} 5 \\
\text { días }\end{array}$ & $\begin{array}{c}\text { Leves a } \\
\text { moderados }\end{array}$ & $\mathrm{IV}+\mathrm{CU}$ & $1 \%$ & $\mathrm{HCQ}+\mathrm{CU}$ & $12 \%$ \\
\hline $\begin{array}{l}\text { Okomus } \\
(2020)^{7}\end{array}$ & Turquía & 60 & Doble ciego & $\begin{array}{l}0,2 \mathrm{mg} / \mathrm{kg}, 5 \\
\text { días }\end{array}$ & Severos & Iv $+C U$ & $20 \%$ & HFA & $30 \%$ \\
\hline
\end{tabular}


Considerando la diversidad de comparaciones, el autor de este comentario efectuó un análisis de sensibilidad limitando el análisis a los dos ECA doble-ciego que comparaban la ivermectina contra placebo (ambas ramas con cuidados usuales no reportados) y el resultado se mantuvo (RR 0,17 [IC95\% 0,06 a 0,48]; $p$ $=0,0008 ; I^{2} 0 \%$; Figura 2).

Los autores reportaron además una recuperación clínica favorable y menor estadía hospitalaria. La ivermectina se asoció con una reducción estadísticamente significativa de los marcadores inflamatorios (proteína $C$ reactiva [9/11 estudios], dímero d [2/6 estudios] y ferritina $3 / 4$ estudios) y un aclaramiento viral más rápido por PCR [8/14 estudios]. El aclaramiento viral fue más pronunciado con dosis más altas de ivermectina $(0,4 \mathrm{mg} / \mathrm{kg})$ hasta por cinco días de tratamiento, alcanzando efectos beneficiosos estadísticamente significativos en los cuatro ECA que utilizaron estos esquemas.

Figura 1. Meta-análisis de ivermectina versus control para el desenlace mortalidad. Notas: IC $95 \%$ : Intervalo de Confianza del $95 \%$. Dominios de la evaluación del riesgo de sesgo: A. Generación aleatoria de la secuencia; B. Enmascaramiento de la asignación; C. Cegamiento de los participantes y del personal; D. Cegamiento de los evaluadores de los desenlaces; E. Datos de resultado incompletos; F. Reporte selectivo de los resultados. Categorías de riesgo de sesgo: en color verde: bajo riesgo de sesgo; rojo: alto riesgo de sesgo; amarillo: riesgo de sesgo incierto o poco claro.

\begin{tabular}{|c|c|c|c|c|c|c|c|c|c|}
\hline \multirow[b]{2}{*}{ Estudio } & \multicolumn{2}{|c|}{ Ivermectina } & \multicolumn{2}{|c|}{ Control } & \multirow[b]{2}{*}{ Peso } & \multirow{2}{*}{$\begin{array}{l}\text { Riesgo Relativo } \\
\text { Random, IC 95\% }\end{array}$} & \multirow{2}{*}{\multicolumn{2}{|c|}{$\begin{array}{c}\text { Riesgo Relativo } \\
\text { Efectos aleatorios, IC } 95 \%\end{array}$}} & \multirow{2}{*}{ Riesgo de sesgo } \\
\hline & Eventos & Total & Eventos & Total & & & & & \\
\hline Hashim 2020 & 2 & 70 & 6 & 70 & $19,9 \%$ & $0,33(0,07$ a 1,60$)$ & $\longrightarrow$ & 干 & \\
\hline Mahmud 2020 & 0 & 183 & 3 & 180 & $5,7 \%$ & $0,14(0,01$ a 2,70$)$ & & & \\
\hline Niaee 2021 & 4 & 120 & 11 & 60 & $24,6 \%$ & $0,18(0,06$ a 0,55$)$ & & & \\
\hline Kirti 2021 & 0 & 55 & 4 & 57 & $5,9 \%$ & $0,12(0,01$ a 2,09$)$ & - & & ??? ? ? ? \\
\hline Elgazzar 2020 & 2 & 200 & 24 & 200 & $18,0 \%$ & $0,08(0,02$ a 0,35$)$ & $\rightarrow$ & - & ? ? ? ? ? ? \\
\hline Okumus 2020 & 6 & 30 & 9 & 30 & $29,8 \%$ & $0,67(0,27$ a 1,64$)$ & & & \\
\hline Total (IC 95\%) & & 658 & & 597 & $100 \%$ & $0,25(0,12$ a 0,52$)$ & 0.0 & 100 & \\
\hline $\begin{array}{l}\text { Eventos totales } \\
\text { Hetoreneidad }\end{array}$ & $\begin{array}{c}14 \\
1^{2}=34 \%\end{array}$ & & 57 & & & & Favorece a ivermectin & Favorece al control & \\
\hline
\end{tabular}

Figura 2. Meta-análisis de ivermectina versus placebo para el desenlace mortalidad. Notas: IC $95 \%$ : Intervalo de Confianza del $95 \%$. Dominios de la evaluación del riesgo de sesgo: A. Generación aleatoria de la secuencia; B. Enmascaramiento de la asignación; C. Cegamiento de los participantes y del personal; D. Cegamiento de los evaluadores de los desenlaces; E. Datos de resultado incompletos; F. Reporte selectivo de los resultados. Categorías de riesgo de sesgo: en color verde: bajo riesgo de sesgo; rojo: alto riesgo de sesgo; amarillo: riesgo de sesgo incierto o poco claro.

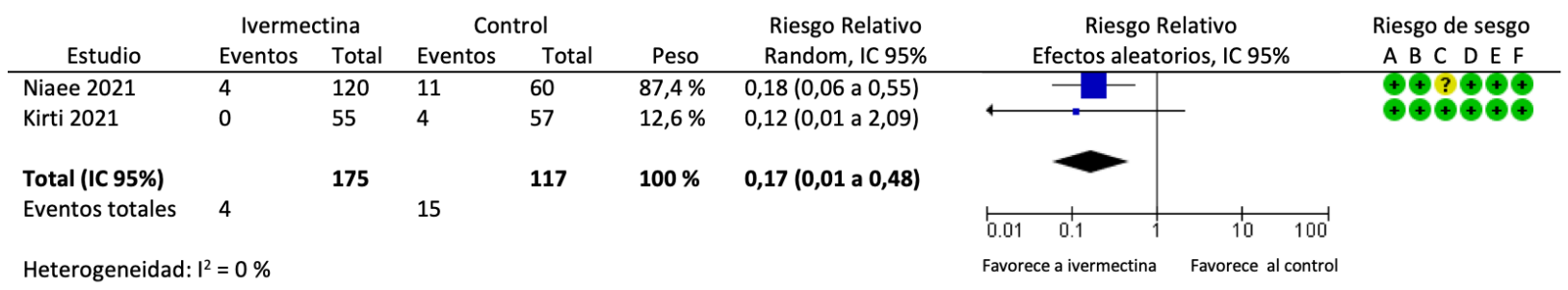

\section{Conclusión}

Se observó una mejora en la sobrevida, tiempo más rápido para la recuperación clínica y sugerencias de un efecto dosisdependiente del aclaramiento viral para los pacientes que reci- bieron ivermectina versus tratamiento de control.

Fuente de financiamiento: UNITAID (iniciativa internacional que promueve el acceso al tratamiento de enfermedades como SIDA, malaria y tuberculosis en países en vías de desarrollo).

\section{Comentario}

La pandemia de SARS-CoV-2 sigue creciendo, acumulando más de 103 millones de casos y 2.241 .409 muertes registradas en todo el mundo al 2 de febrero de $2021^{8}$. Los suministros vacunas aún son escasos como para cubrir oportunamente las necesidades globales. En consecuencia, investigadores de todo el mundo están buscando con urgencia intervenciones, nuevas o ya existentes, para prevenir nuevas infecciones o la progresión de la enfermedad en quienes ya están infectados. En una situación de pandemia, los perfiles de seguridad conocidos, el menor tiempo de desarrollo, los menores precios y 
la mayor capacidad de escalar la producción, hace a los fármacos ya existentes particularmente atractivos, en comparación con el descubrimiento de nuevos fármacos.

Tres fármacos han mostrado beneficios en la sobrevida significativos a la fecha: la dexametasona en el ensayo RECO$\mathrm{VERY}^{9,10}$ para pacientes con indicación de oxígeno, el tocilizumab y el sarilumab, en el Ensayo REMAP-CAP ${ }^{11}$ para pacientes en cuidados intensivos. Otros como la hidroxicloroquina, la combinación lopinavir/ritonavir, el remdesivir y el interferón beta, no han demostrado beneficios en la supervivencia en dos grandes ECAs ${ }^{10,12}$.

La ivermectina es un fármaco antiparasitario bien establecido con actividad inmunomoduladora y antiinflamatoria, no autorizado para mujeres embarazadas o en período de lactancia, ni para niños de menos de $15 \mathrm{~kg}$ de peso. A dosis estándar, de 0,2 a $0,4 \mathrm{mg} / \mathrm{kg}$ durante 102 días, la ivermectina tiene un buen perfil de seguridad y se ha administrado a miles de millones de personas en todo el mundo. Un meta-análisis reciente no encontró diferencias importantes en los eventos adversos en los que recibieron dosis más altas de ivermectina, de hasta $2 \mathrm{mg} / \mathrm{kg}$, y en los que recibieron ciclos más largos, de hasta 4 días, en comparación con los que recibieron dosis estándar ${ }^{13}$.

La revisión sistemática resumida en este artículo -reporte que aún no fue evaluado por pares y se encuentra disponible en internet con acceso abierto a través de un repositorio de pre-impresiones- sugiere que el tratamiento con ivermectina reduce los marcadores inflamatorios, logra más rápidamente la supresión viral y, lo más prometedor, mejora la sobrevida de los pacientes con COVID-19 en comparación con los cuidados usuales. Sin embargo, estos resultados deben interpretarse con cautela en virtud de varias limitaciones.

En primer lugar es necesario tener en cuenta las limitaciones vinculadas a la conducción de esta revisión, evaluada mediante los 16 ítems de la herramienta AMSTAR-2 ${ }^{14,15}$. Tras aplicar este instrumento, se observa que en siete items se cumplen las expectativas metodológicas (en dos de ellos, parcialmente), mientras que en nueve de ellos, no se cumplen los requisitos. Cuatro de los items calificados negativamente forman parte de los dominios considerados críticos: protocolo registrado antes de la revisión, justificación de los estudios excluidos, consideración del riesgo de sesgo en la interpretación de los resultados de la revisión, evaluación de la presencia y el impacto probable del sesgo de publicación; lo que resulta en una confianza global de la revisión sistemática críticamente baja (ver lista de cotejo AMSTAR-2 en material suplementario).

En segundo término, también hay limitaciones importantes en cuanto a la certeza de la evidencia que se sintetiza en esta revisión sistemática, que puede ser evaluada mediante el abordaje GRADE ${ }^{16}$. En este caso, se parte de alta calidad de evidencia por tratarse de ECA. Enfocándonos en el desenlace más crítico, como lo es la mortalidad, a continuación evaluaremos los cinco dominios que pueden reducir la certeza en la reducción del $75 \%$ de la mortalidad estimada para la ivermectina.

1. Limitaciones metodológicas de los estudios (riesgo de sesgo). Para los estudios abiertos, existe un riesgo de sesgo en la evaluación de criterios de valoración subjetivos, como la recuperación clínica y el alta hospitalaria. Sin embargo, el riesgo es menor para desenlaces objetivos, como la supresión viral y la mortalidad. Como puede apreciarse en la Figura 1, este cuerpo de la evidencia merece al menos la reducción un nivel, pasando de alta a moderada certeza de la evidencia. Además, muchos de los estudios evaluados no han sido revisados por pares y la deficiente calidad de reporte y conducción podría evidenciarse aún más tras un adecuado proceso editorial.

2. Inconsistencia en los resultados. Si bien la heterogeneidad en términos estadísticos es de baja a moderada $\left(I^{2}\right.$ $>30 \%$ ), se pueden detectar fuentes de heterogeneidad en aspectos metodológicos (ECA de simple o doble-ciego y abiertos), en las poblaciones (desde COVID-19 sintomáticos a casos severos, con tasas de mortalidad en un rango entre 2 y $30 \%$ también explicado por la amplia gama de países, a menudo en condiciones de recursos y sistemas de salud sobrecargados), en las intervenciones (estudios que difieren en dosis, duración del tratamiento, en combinaciones que incluyen cuidados usuales no descriptos o doxiciclina y en comparaciones con fármacos no recomendados, como la hidroxicloroquina u otros antimicrobianos) y en la incertidumbre en cuanto del tiempo de medición de los desenlaces críticos. Las medidas de resultado virológico no se estandarizaron y se midieron en diferentes puntos de tiempo y con diferentes umbrales de PCR. Por estos motivos, debieramos descender otro nivel, pasando de moderada a baja certeza de la evidencia.

3. Evidencia indirecta. Dado que en los estudios se evaluó en forma directa la mortalidad, no hay problemas con este dominio.

4. Imprecisión. En apariencia no hay problemas con este dominio; dado que en ambos extremos del IC $95 \%$ se observan impactantes reducciones que van del 48 al $88 \%$ en la mortalidad, las decisiones no cambiarían ni aun en el peor de los escenarios, en el que representaría bajar la mortalidad por COVID-19 casi a la mitad. Sin embargo, al estudiarse pocos eventos aunque el intervalo de confianza sea estrecho, podría suceder que el tamaño (muestral) óptimo de la información (TOI u OIS, por sus iniciales en inglés) sea insuficiente pues resulta inestable. Para demostrar con precisión una reducción del $75 \%$ en la mortalidad, el TOI es de 98 participantes para una mortalidad en el grupo control de $30 \%{ }^{7}$ pero llega a 1.720 participantes para una mortalidad en el grupo control de $2 \%{ }^{3}$. Considerando el extremo del IC $95 \%$ del efecto (RR de $48 \%$ ) las cifras van de 282 a 4.636, respectivamente. Dado que para el desenlace mortalidad sólo se reúnen 1.255 participantes no se cumple el criterio TOI en todos los casos, y debemos bajar un nivel más en la certeza de la evidencia, que pasa de baja a muy baja.

5. Sesgo de publicación (publicación selectiva de los ensayos con resultados "positivos", dejando sin publicar los "negativos"). Aunque estadísticamente no es posible evaluarlo, cuando se dispone de menos de 10 estudios en un meta-análisis, existen preocupaciones por fuera del análisis estadístico que sugieren con fuerza la posibilidad de este sesgo verdaderamente crítico, sobre todo en contexto de pandemia. Un búsqueda rápida en Epistemonikos (base de datos colaborativa multilingüe de evidencia en salud) identificó a la fecha al menos cuatro ensayos más que los incluidos en esta revisión. Por ejemplo, el ECA colombiano EPIC15 que no tuvo resultados positivos según la 
conferencia de prensa el 30 de diciembre ${ }^{17}$. Se trata del ensayo más grande realizado hasta ahora (400 participantes analizados de los 476 pacientes planificados) pero como los datos aún no se han dado conocer completamente, no es posible combinar sus datos con el cuerpo de la evidencia existente. En esta revisión, aunque se contactó a cada equipo de investigación que realiza los ensayos, no se buscó en la literatura gris (literatura no indizada) por lo que puede haber más estudios negativos cómo el EPIC, por lo que la estimación del impacto de la ivermectina en la mortalidad podría estar sobrevalorado. Por este motivo deberíamos bajar un nivel la certeza de la evidencia, que ya era muy baja.

En definitiva, aunque el efecto de la ivermectina parezca contundente, la muy baja certeza de la evidencia en cuanto a la mortalidad no permite hacer recomendaciones. Los ECA más grandes actualmente en curso en México, Sudamérica y Egipto, con resultados esperados en febrero y marzo de 2021, podrían mejorar esta certeza de la evidencia.

A pesar de las limitaciones mencionadas, el presente estudio sugiere un impacto potencial que podría depender de la dosis y la duración de ivermectina sobre la tasa de supresión viral. La dosis máxima eficaz de ivermectina aún no está clara y los nuevos ensayos clínicos están evaluando dosis más altas, de hasta $1,2 \mathrm{mg} / \mathrm{kg}$ durante 5 días. Sin embargo los datos invitro actuales no apoyan una actividad antiviral directa de la droga.

El beneficio de supervivencia del $75 \%$ observado en este meta-análisis se basa solo en 71 muertes, en seis ensayos clínicos diferentes. Este número total de muertes es menor que en los ensayos RECOVERY ${ }^{9}$ o REMAP-CAP ${ }^{11}$, que llevaron a la aprobación de la dexametasona, el tocilizumab y el sarilumab. Sin embargo, el gran beneficio potencial de sobrevida, más fuerte que para las otras drogas reutilizadas, junto a la evidencia indirecta de mejoría de los marcadores inflamatorios y de la supresión viral, hacen imperativo confirmar o rechazar esta alentadora posibilidad mediante futuras investigaciones de alta calidad, aclarando la dosis y la duración de tratamiento óptima de la ivermectina para diferentes poblaciones.

Es posible que la conclusiones vertidas en este comentario, tras un análisis crítico de la evidencia, generen automáticamente rechazos en algunos sectores de la sociedad. Sobre todo si tenemos en cuenta que varios países latinoamericanos como Guatemala, Bolivia, Perú, Honduras, Brasil y México usan esta estrategia terapéutica contra el COVID-19. La utilización de la ivermectina sigue ganando adeptos en la Argentina, y esta semana la provincia de Misiones aprobó su utilización para pacientes con coronavirus, a la vez que las de Tucumán, Salta y Corrientes ya lo han adoptado de manera oficial, y las de La Pampa y Chaco la están utilizando pese a no tener autorización de la Administración Nacional de Medicamentos, Alimentos y Tecnología Médica (ANMAT). De manera coincidente, la agencia a cargo de aprobar el uso de medicamentos en los EE.UU. (FDA, por sus iniciales en inglés), informó que se necesitan pruebas adicionales para determinar si la ivermectina puede ser apropiada para prevenir o tratar el coronavirus y la Organización Mundial de la Salud (OMS) directamente no lo recomienda como tratamiento para COVID-19.

Sería bueno recordar casos como el de la cloroquina o del dióxido de cloro, que se anunciaron en los medios con bombos y platillos, generando gran expectativa en la población, entusiasmada por su accesibilidad, pero con consecuencias eventualmente muy serias.

\section{Conclusiones del comentador}

En el momento de redactar este artículo, las brechas de la evidencia impiden una conclusión sólida sobre la eficacia clínica de la ivermectina en el tratamiento de pacientes con COVID-19. La eficacia de esta intervención debe validarse en ECA grandes y metodológicamente adecuados antes de que las autoridades reguladoras aprueben su uso, ya que aún no podemos asegurar con certeza que los beneficios sean mayores que los riesgos y los costos.

Agustín Ciapponi [ Centro Cochrane Argentina, Instituto de Efectividad Clínica y Sanitaria; Servicio de Medicina Familiar y Comunitaria, Hospital Italiano de Buenos Aires. agustin.ciapponi@hospitalitaliano.org.ar ]

Ciapponi A. Meta-análisis preliminar de ensayos clínicos aleatorizados de ivermectina para tratar la infección por SARS-CoV-2. Evid Actual Pract Ambul. 2021;24(1):e002117. Comentado de: Hill A, et al. Meta-analysis of randomized trials of ivermectin to treat SARS-CoV-2 infection. Research Square 2021:10.21203/rs.21203.rs-114482/v114484

\section{Referencias}

1. Hill A, Abdulamir A, Ahmed S, et al. Meta-analysis of randomized trials of ivermectin to treat SARS-CoV-2 infection. Research Square. 2021;Available from: 10.21203/rs.3.rs-148845/v1.

2. Hashim HA, Maulood MF, Rasheed AM, et al. Controlled randomized clinical trial on using Ivermectin with Doxycycline for treating COVID-19 patients in Baghdad, Iraq. medRxiv. 2020;Available from: 10.1101/2020.10.26.20219345.

3. Mahmud R. Clinical Trial of Ivermectin Plus Doxycycline for the Treatment of Confirmed Covid-19 Infection; 2020 . Available from: https://clinicaltrials. gov/ct2/show/results/NCT04523831?view=results

4. Niaee M, Gheibi N, Namdar P, et al. Ivermectin as an adjunct treatment for hospitalized adult COVID-19 patients: A randomized multi-center clinical trial. Reaserch Square. 2020;Available from: 10.21203/rs.3.rs-109670/v1.

5. Ravirkirti, Roy R, Pattadar C, et al. Ivermectin as a potential treatment for mild to moderate COVID-19 - A double blind randomized placebo-controlled trial. medRxiv. 2021;Available from: 10.1101/2021.01.05.21249310.

6. Elgazzar A, Hany B, Youssef S, et al. Efficacy and safety of ivermectin for treatment and prophylaxis of COVID-19 pandemic. ResearchSquare. 2020; Available from: 10.21203/rs.3.rs-100956/v2.

7. Okumuşs N. Ivermectin for Severe COVID-19 Management; 2021. Available from: https://ClinicalTrials.gov/show/NCT04646109;2020 [Last access: 2021-02-02].

8. COVID-19 Map - Johns Hopkins Coronavirus Resource Center; 2021. Available from: https://coronavirus.jhu.edu/map.html [Last access: 2021-0202] 
9. Group RC, Horby P, Lim WS, et al. Dexamethasone in Hospitalized Patients with Covid-19 - Preliminary Report. N Engl J Med. 2020;p. NEJMoa2021436. Available from: 10.1056/NEJMoa2021436.

10. Ciapponi A. La dexametasona redujo la mortalidad de pacientes COVID-19 en ventilación mecánica invasiva u oxigenoterapia. Comentado de: Horby P, et al. Effect of Dexamethasone in Hospitalized Patients with COVID-19 - Preliminary Report. medRxiv 2020. doi:10.1101/2020.06.22.20137273 . Evid Actual Pract Ambul. 2020;23(3):e002078. Available from: http://www.evidencia.org/index.php/Evidencia/article/view/6867.

11. Gordon AC, Mouncey PR, Al-Beidh F, et al. Interleukin-6 Receptor Antagonists in Critically III Patients with Covid-19 - Preliminary report. medRxiv. 2001; Available from: 10.1101/2021.01.07.21249390.

12. Paul SS, Biswas G. Repurposed antiviral drugs for COVID-19 -interim WHO SOLIDARITY trial results. Mini Rev Med Chem. 2020;Available from: $10.2174 / 1389557521666201222145842$

13. Navarro M, Camprubi D, Requena-Mendez A, et al. Safety of high-dose ivermectin: a systematic review and meta-analysis. J Antimicrob Chemother. 2020;75(4):827-834. Available from: 10.1093/jac/dkz524.

14. Shea BJ, Reeves BC, Wells G, et al. AMSTAR 2: a critical appraisal tool for systematic reviews that include randomised or non-randomised studies of healthcare interventions, or both. BMJ. 2017;358:j4008. Available from: 10.1136/bmj.j4008.

15. A C. AMSTAR-2: herramienta de evaluación crítica de revisiones sistemáticas de estudios de intervenciones de salud. Traducido, resumido y comentado de: Shea BJ, y col. AMSTAR 2: a critical appraisal tool for systematic reviews that include randomised or non-randomised studies of healthcare interventions, or both. BMJ. 2017; 358.j4008. PMID: 28935701 [PubMed - indexed for MED- LINE] PMCID: PMC5833365. 2018;21(1):413. Available from: http://www.evidencia.org.ar/index.php/Evidencia/article/view/6834.

16. Guyatt G, Oxman AD, Akl EA, et al. GRADE guidelines: 1. Introduction-GRADE evidence profiles and summary of findings tables. J Clin Epidemiol. 2011;64(4):383-394. Available from: 10.1016/j.jclinepi.2010.04.026.

17. López-Medina E. Efficacy of Ivermectin in Adult Patients With Early Stages of COVID-19 (EPIC Trial); 2020. Available from: https://ClinicalTrials.gov/ show/NCT04405843;2020 [Last access: 2021-02-02]. 